\title{
Brownian modulated optical nanoprobes
}

\author{
C. J. Behrend, J. N. Anker, and R. Kopelman \\ Department of Chemistry, University of Michigan, Ann Arbor, Michigan 48109-1055
}

(Received 13 August 2003; accepted 9 November 2003)

\begin{abstract}
Brownian modulated optical nanoprobes (Brownian MOONs) are fluorescent micro- and nanoparticles that resemble moons: one hemisphere emits a bright fluorescent signal, while an opaque metal darkens the other hemisphere. Brownian motion causes the particles to tumble and blink erratically as they rotate literally through the phases of the moon. The fluctuating probe signals are separated from optical and electronic backgrounds using principal components analysis or images analysis. Brownian MOONs enable microrheological measurements on size scales and timescales that are difficult to study with other methods. Local chemical concentrations can be measured simultaneously, using spectral characteristics of indicator dyes embedded within the MOONs. (C) 2004 American Institute of Physics. [DOI: 10.1063/1.1637963]
\end{abstract}

Rotational viscosity is a fundamental property of materials relevant to fields from biophysics to materials and chemical processing. Its measurement is important for understanding molecular behavior, cell function, colloidal properties, and bulk materials. For non-Newtonian and heterogeneous fluids, the measured viscosity is a complex function of timescales over which forces are applied, and the size scale of the instrument used to probe the viscosity. ${ }^{1}$ On the microscopic scale, measurements of viscosity in the cell cytosol have varied over six orders of magnitude depending on the size of the probe used. ${ }^{2}$ Recent work suggests sieve-like structures within subdomains of cytoplasm that allow small particles to diffuse through while resisting the motion of larger particles. ${ }^{3}$ In addition, liquid filled structures such as endosomes may allow particles to rotate within but not translate, causing divergence between rotational and translational Brownian diffusion. Macroscopic instruments determine viscosity by measuring the resistance of a fluid to mechanical movement. Similarly, thermal fluctuations of microscopic particles can be used to mechanically probe viscosity on a much finer scale.

In 1828, Robert Brown first observed pollen grains translating in unpredictable directions and tumbling around their own axes. ${ }^{4,5}$ This "Brownian motion" was explained in 1906 by Albert Einstein who derived the following equations for the rotational and translational diffusion coefficient, $D_{0}^{\text {rot }}$ and $D_{0}^{\text {trans }}$ of a spherical particle: ${ }^{6}$

$$
D_{0}^{\mathrm{rot}}=\frac{k_{B} T}{8 \pi \eta r^{3}}, \quad D_{0}^{\mathrm{trans}}=\frac{k_{B} T}{6 \pi \eta r},
$$

where $k_{B}$ is Boltzmann's constant, $T$ is the temperature, $\eta$ is the viscosity, and $r$ is the particle radius. Jean Perrin employed studies of Brownian motion of $13 \mu \mathrm{m}$ particles to demonstrate the existence of molecules and evaluate Avogadro's number. ${ }^{7}$ Most current studies of microsphere rotation continue to use larger particles $(>2 \mu \mathrm{m})$ with observable landmarks.

Molecular translational and rotational diffusion is studied by optical techniques such as fluorescence anisotropy, fluorescence recovery after photobleaching (FRAP), and fluorescence correlation spectroscopy. ${ }^{8}$ To measure transla- tional diffusion on larger size scales $(50 \mathrm{~nm}-5 \mu \mathrm{m})$, the motion of nanospheres and microspheres are observed while they are pulled through the solution with magnetic tweezers, ${ }^{9}$ or move under the influence of Brownian forces. ${ }^{10}$ Rotational motion of microspheres and nanospheres has proven more difficult to measure. Dynamic light scattering and polarized FRAP are used to determine rotational diffusion coefficients for ensembles of particles; however, these techniques generally acquire an average over a large number of particles and are effected by scattering. ${ }^{11}$ Alternatively, particle orientation may be observed directly, but only if each particle has an observable landmark ${ }^{7,12}$ or is not spherical in shape. ${ }^{13}$ Particle orientation may also be determined by measuring the average magnetic moment from magnetized particles. ${ }^{2}$ However, the magnetic methods provide information only on the average particle and require a relatively large number of magnetic particles to obtain an accurate reading. ${ }^{14}$

Brownian MOONs ${ }^{15}$ provide a method for measuring rotational viscosity in the vicinity of single particles. Brownian MOONs have one hemisphere coated with an opaque metal cap so that only the other hemisphere is able to emit light. The particles blink erratically as Brownian motion causes them to tumble. Fluorescent detection of this modulated signal allows the dynamics affecting small particles to be observed, with high accuracy and on short timescales. A simple process is used to prepare large batches of particles, and their size can be controlled over a wide range, including particles far smaller than the diffraction limit of visible light.

The autocorrelation function of the intensity fluctuations from a randomly rotating Brownian MOON provides a measure of the rotational diffusion rate. Experiments using magnetic fields to rotate $4.4 \mu \mathrm{m}$ magnetically modulated optical nanoprobes (MagMOONs) indicated the fluorescence intensity is proportional to the cosine of the azimuthal angle of the particles, with weaker harmonics also appearing. ${ }^{15}$ Assuming that the fluorescence intensity from Brownian MOONs is also proportional to the cosine of the azimuthal angle, the autocorrelation function, $G^{\text {rot }}(\tau)$, of the fluctuations is expected to decay exponentially [Eq. (2)]: ${ }^{16}$

$$
G^{\mathrm{rot}}(\tau)=e^{-t / \tau}, \quad \tau=\frac{\kappa V \eta}{2 k_{B} T} .
$$




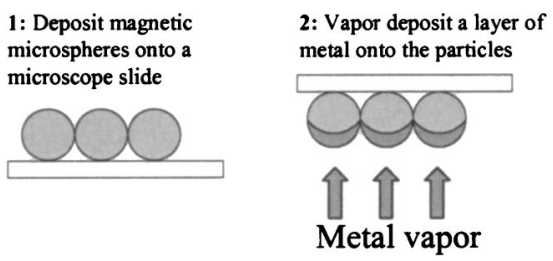

3: Remove particles from glass slide using an artist's paint brush + sonicate

FIG. 1. Schematic of a method to prepare Brownian MOONs.

For a spherical particle, the shape factor $\kappa$ is 6 ; for aspherical particles, the shape factor depends on the relative dimensions of the particle. The rotational diffusion time depends on volume, $V$, of the particle and is proportional to the viscosity of the surrounding solvent environment. If harmonics are present in the angular intensity distribution, the autocorrelation function is expected to decay more quickly.

Brownian MOONs are produced using simple physical processes. Fluorescent nano- or microspheres are allowed to settle, forming a monolayer on a microscope slide. Using vapor deposition of aluminum, or sputter-coated gold, one hemisphere of the particles is coated with around $100 \mathrm{~nm}$ thick layer of an opaque metal, as shown in Fig. 1. This metal capping layer prevents excitation light from reaching the fluorophores and also blocks emitted fluorescence from leaving through the coated hemisphere of the particle. After completing the coating, the particles are removed from the glass slide by gentle brushing with an artists brush. Sonicating the brush at $42 \mathrm{kHz}$ for $20 \mathrm{~s}$ in aqueous solution suspends the particles. We have prepared Brownian MOONs from microspheres and nanospheres that range in diameter from 200 $\mathrm{nm}$ to $4.4 \mu \mathrm{m}$. The $2 \mu \mathrm{m}$ and $850 \mathrm{~nm}$ fluorescent particles were purchased from Spherotech (Libertyville, IL), and the 300 and $200 \mathrm{~nm}$ particles were purchased from Bangs Labs (Fishers, IN). Changing the coating thickness allows fine control over the asphericity of the Brownian MOONs. A coating thickness of $\sim 20 \mathrm{~nm}$ is sufficient to render the particles opaque, and even a $10 \mathrm{~nm}$ coating will produce a modulated signal. ${ }^{17}$

Figure 2(a) shows an image of $2 \mu \mathrm{m}$ Brownian MOONs in different orientations (phases of the moon). $2 \mathrm{~s}$ later [Fig. 2(b)], the particles have reoriented due to Brownian forces $(\tau$ is expected to be $2 \mathrm{~s}$ for a $2 \mu \mathrm{m}$ particle in water). The

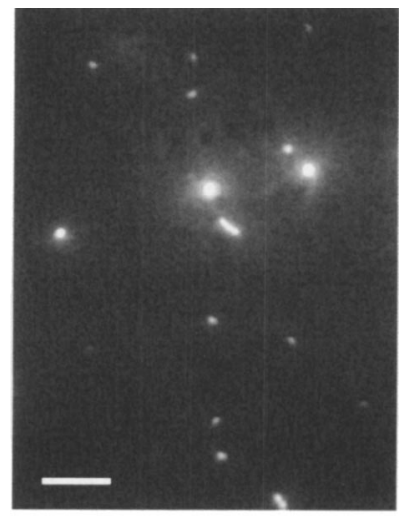

a

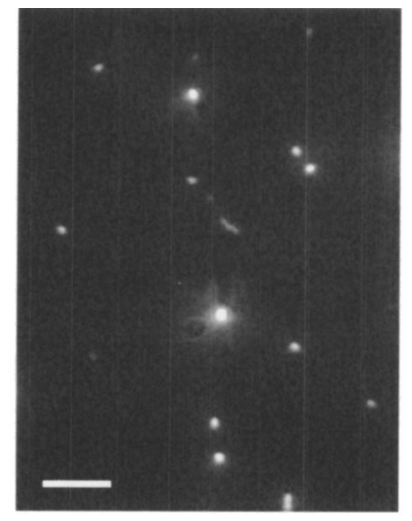

b
FIG. 2. (a) Image of $2 \mu \mathrm{m}$ Brownian MOONs in different orientations (phases of the moon); (b) $2 \mathrm{~s}$ later, the particles have reoriented due to Brownian forces. Scale bars are $20 \mu \mathrm{m}$ (also see EPAPS Ref. 18). crescent shape is clear for $2 \mu \mathrm{m}$ particles, but particles smaller than the diffraction limit only change intensity but not apparent shape as they rotate. Two small chains are present in the center and bottom of Fig. 2. The orientation of these chains can be observed around three axes of the particle, allowing complete characterization of the motion. A low number of such chains are formed during the coating process, and they can be dispersed with sonication.

Brownian MOONs were suspended in glycerol-water solutions with different viscosities and put into a demountable $100 \mu \mathrm{m}$ quartz sample chamber (Starna, Atascadero, CA). The small thickness of this cell prevented convection from affecting the particle motion. The particles were viewed with an Olympus IMT-II (Lake Success, NY) inverted fluorescence microscope. Fluorescence spectra from Brownian MOONs were acquired using an Acton Research Corp. spectrograph and a Hamamatsu HC230 CCD interfaced with an Intel Pentium computer. Images and videos (see EPAPS Ref. 18) of Brownian MOONs were acquired with a Nikon Coolpix 995 camera. Single particles were observed with a $40 \times$ objective. It was clear by inspection that the rate of reorientation and blinking was slower for MOONs in higher viscosity solutions and for larger particles. A time-series of spectra were taken of single $850 \mathrm{~nm}$ particles reorienting under Brownian motion. Usual data sets consisted of from 200 to 1000 spectra, recorded at 100 or $200 \mathrm{~ms}$ integration times, with no programmed delay between spectral acquisitions. Principal components analysis (PCA) was applied to the time series in order to separate the Brownian MOON signal from other signals present in the spectra. In order to determine the rate of rotational diffusion, the autocorrelation function was calculated for the time series of the Brownian MOON fluctuations.

During the PCA, signals from Brownian MOONs are separated from the background, scattering, or other sources of fluorescence that may have an overlapping spectral emission. This signal separation dramatically improves signal to background levels. The main sources of light contributing to the observed spectra were determined using PCA and are shown in Fig. 3(b). The time series, or "score," for each of the components in Fig. 3(c) is calculated by projection of the principal components onto the original data space. ${ }^{19,20}$ The random fluctuation rate for the Brownian MOONs, with a characteristic correlation time, $\tau$, is directly proportional to the rotational viscosity. Brownian MOONs thus provide a measurement of viscosity in their local environments.

The autocorrelation time for $850 \mathrm{~nm}$ aluminum coated Brownian MOONs in each solution was measured and used to prepare the calibration curve shown in Fig. 4. Figure 4(a) shows typical autocorrelation functions from particles in different viscosity liquids. Figure 4(b) shows a calibration curve from the average autocorrelations for a number of particles and trials. The autocorrelation function decayed exponentially and, as expected, the decay rate increased linearly with increasing viscosity. Overall, the observed values for $\tau$ were systematically $25 \%$ shorter than predicted from theory. The theoretical values were calculated using Eq. (2) and included the volume change from the $100 \mathrm{~nm}$ aluminum capping (which increases $\tau$ by 18\%) and the aspherical shape factor (which increased $\tau$ by $5 \%$ ). Additional sources of error in- 


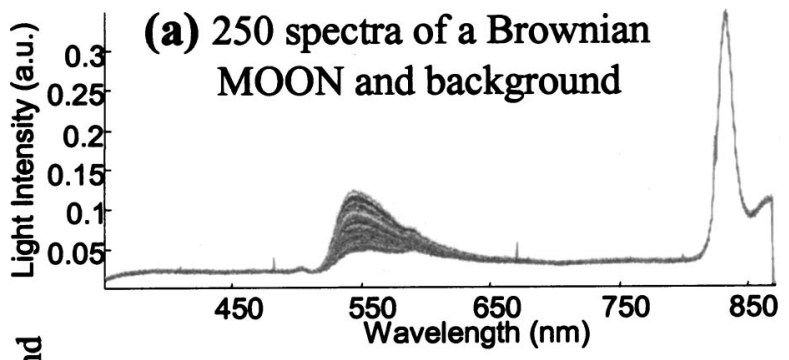

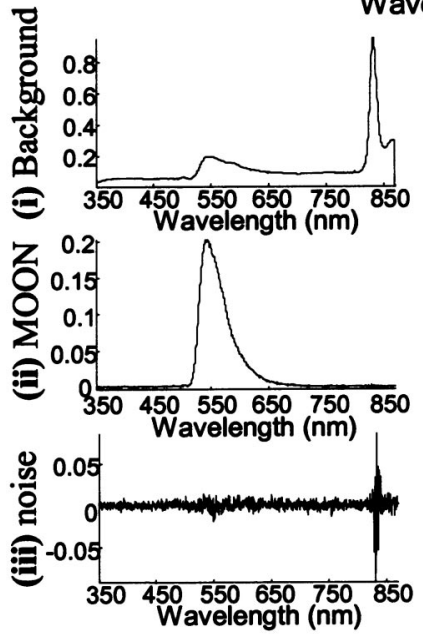

(b)
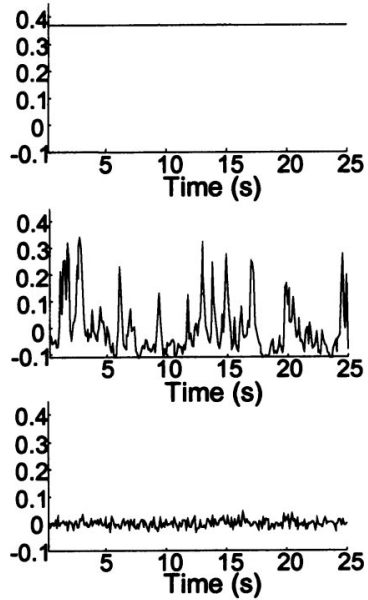

(c)

FIG. 3. (a) Time series of 250 spectra containing multiple signals that vary in time. Shown in (b) are the spectra from the PCA with their corresponding time series shown in (c). Underlying spectra sources include (i) background fluorescence and mercury lamp reflection, (ii) Brownian MOON spectrum, (iii) noise.

clude the presence of harmonics in the intensity dependence on angle which would decrease the observed $\tau$. In addition, the viscosity of glycerol depends strongly on temperature and concentration, particularly at high glycerol concentration. At $68 \%$ glycerol and $24{ }^{\circ} \mathrm{C}$, a $1 \%$ change in glycerol concentration changes the viscosity by $10 \%$, and an increase in temperature of $1{ }^{\circ} \mathrm{C}$ decreases viscosity by $5 \% .^{21}$ A $100 \mathrm{~ms}$ maximum acquisition rate for the spectrometer also limited our ability to detect fast changes in the fluorescence intensity at lower viscosities.

In addition to measuring viscosity random rotation of the Brownian MOON modulates its fluorescence so that its spectrum is distinguishable from that of a constant background or signal sources that have different time signatures. PCA is

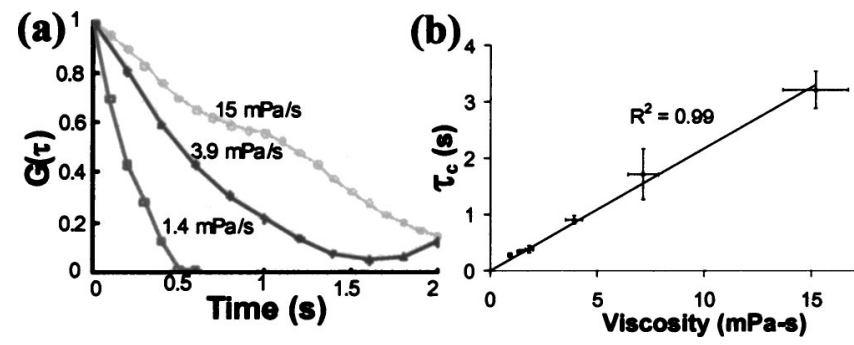

FIG. 4. (a) Typical autocorrelation functions of the time series for particles in different viscosity solutions; (b) average decay time, $\tau$, of the autocorrelation function measured for a series of viscosities. The $y$-axis errors show the standard error for $\langle\tau\rangle$, while the $x$-axis error bars show a $10 \%$ error estimated from variation in particle volume and temperature variation. Viscosity was calculated from glycerol composition at $24^{\circ} \mathrm{C}$ (Ref. 21). used to separate the particle's genuine spectrum from other signal sources [Fig. 3(b)]. This spectral separation promises to improve the detection limits for already highly sensitive chemical tests, based on spectral shapes of indicator dyes and labels, while simultaneously sampling the local physical environment. Existing chemical sensors for ratiometric intracellular imaging of ions, dissolved gases, and small molecules such as glucose, may be simply transformed into Brownian MOONs by capping them with metal. ${ }^{22-25}$

In summary, Brownian MOONs function as nanoviscometers by modulating the fluorescent signal at different characteristic rates in solutions of varying viscosity. The unique time signature generated by the random Brownian motion allows separation of signal from background. These MOONs also promise improvement of sensitivity and reduction of complexity for current immunoassay procedures and for intracellular chemical sensors. ${ }^{16,26,27}$

The authors gratefully acknowledge support from NSF Grant No. DMR 9900434.

${ }^{1}$ F. C. Mackintosh and C. F. Schmidt, Curr. Opin. Colloid Interface Sci. 4, 300 (1999).

${ }^{2}$ P. A. Valberg and H. A. Feldman, Biophys. J. 52, 551 (1987).

${ }^{3}$ L. W. Janson, K. Ragsdale, and K. Lubyphelps, Biophys. J. 71, 1228 (1996).

${ }^{4}$ R. Brown, Edinburgh Philos. J. 5, 358 (1828).

${ }^{5}$ R. Brown, Edinburgh J. Sci. 1, 314 (1829).

${ }^{6}$ A. Einstein, Investigations on the Theory of the Brownian Movement (Dover, New York, 1956).

${ }^{7}$ J. Perrin, Brownian Movement and Molecular Reality (Taylor and Francis, London, 1910).

${ }^{8}$ J. R. Lakowicz, Principles of Fluorescence Spectroscopy (Plenum, New York, 1983).

${ }^{9}$ F. H. C. Crick and A. F. W. Hughes (1950), Vol. 37

${ }^{10}$ T. Gisler and D. A. Weitz, Curr. Opin. Colloid Interface Sci. 3, 586 (1998).

${ }^{11}$ G. H. Koenderink, Rotational and Translational Diffusion in Colloidal Mixtures (Cip-Gegevens Koninklikjke Bibliotheek, Dan Haag, Netherlands, 2003)

${ }^{12}$ G. Romano, L. Sacconi, M. Capitanio, and F. S. Pavone, Opt. Commun. 215, 323 (2003).

${ }^{13}$ R. Yasuda, H. Miyata, and K. Kinosita, J. Mol. Biol. 263, 227 (1996).

${ }^{14}$ W. Moller, T. Hofer, A. Ziesenis, E. Karg, and J. Heyder, Toxicol. Appl. Pharmacol. 182, 197 (2002).

${ }^{15}$ J. N. Anker and R. Kopelman, Appl. Phys. Lett. 82, 1102 (2003).

${ }^{16}$ P. A. Valberg and J. P. Butler, Biophys. J. 52, 537 (1987).

${ }^{17}$ M. Born and E. Wolf, Principles of Optics, 6th ed. (Cambridge University Press, New York, 1980), Chap. 13.

${ }^{18}$ See EPAPS Document No. E-APPLAB-84-006401 for a video of Brownian MOONs. A direct link to this document may be found in the online article's HTML reference section. The document may also be reached via the EPAPS homepage (http://www.aip.org/pubservs/epaps.html) or from ftp.aip.org in the directory/epaps/; see the EPAPS homepage for more information.

${ }^{19}$ A. Carden, R. M. Rajachar, M. D. Morris, and D. H. Kohn, Calcif. Tissue Int. 72, 166 (2003)

${ }^{20}$ R. Tauler, A. Smilde, and B. Kowalski, J. Chemom. 9, 31 (1995).

${ }^{21}$ P. N. Shankar and M. Kumar, Proc. R. Soc. London, Ser. A 444, 573 (1994).

${ }^{22}$ J. W. Aylott, Analyst (Cambridge, U.K.) 128, 309 (2003).

${ }^{23}$ E. J. Park, M. Brasuel, C. Behrend, M. A. Philbert, and R. Kopelman, Anal. Chem. 75, 3784 (2003).

${ }^{24}$ H. Xu, J. W. Aylott, R. Kopelman, T. J. Miller, and M. A. Philbert, Anal. Chem. 73, 4124 (2001).

${ }^{25}$ M. Brasuel, R. Kopelman, T. J. Miller, R. Tjalkens, and M. A. Philbert, Anal. Chem. 73, 2221 (2001).

${ }^{26}$ J. N. Anker, C. Behrend, and R. Kopelman, JACS Commun. (to be published).

${ }^{27}$ J. N. Anker, C. Behrend, and R. Kopelman, J. Appl. Phys. 93, 6698 (2003). 